\title{
Maximum Number of Finite Transmission Zeros of Coupled Resonator Filters With Source/Load-Multiresonator Coupling and a Given Topology
}

\author{
Smain Amari and Jens Bornemann \\ Department of Electrical and Computer Engineering, University of Victoria, BOX 3055 \\ Victoria, B.C. Canada V8W 3P6 \\ E-mail: samari@engr.uvic.ca
}

\begin{abstract}
This paper presents an algorithm to determine the maximum number of finite transmission zeros which can be achieved by a network of $n$ coupled resonators with a fixed topology matrix when the source or the load is coupled to more than one resonator. These zeros can be real, imaginary or complex.
\end{abstract}

\section{Introduction}

Modern communication systems often require filtering structures which can only be achieved by those systems exhibiting transmission zeros at finite frequencies. The resulting sharp cutoff between the different communication channels is essential in avoiding cross-talk. Furthermore, the proximity of these channels is best handled by filters with asymmetrically prescribed transmission zeros [1].

A network of $n$ resonators with additional couplings between non-adjacent resonators is often used to implement these filtering functions. It is well known that a maximum of $\mathrm{n}-2$ transmission zeros can be achieved when the first resonator, which is connected to the source, is also cross-coupled to resonator $\mathrm{n}$ which is connected to the load [2]. An algorithm to determine the maximum number of finite transmission zeros of coupled resonator filters with an arbitrary topology under this excitation scheme was recently presented by Amari [3]. For more stringent specifications, it becomes necessary to generate more transmission zeros by coupling either or both of the source and the load to more than one resonator. A third order filter with the source coupled to two resonators was presented in [4]. This additional coupling allows the generation of 2 finite transmission zeros instead of one. A two-resonator filter with two transmission zeros was given in [5]. The presence of coupling between the load and the source is responsible for the generation of the two finite transmission zeros. Other filters where the number of transmission zeros exceeds $n-2$ were also reported [6-8].

In light of the increasing importance and interest in configurations where the source/load-multiresonator coupling is present, it is important to establish rigorous algorithms to determine the anticipated performance of a given topology. Therefore, this paper focuses on the extension and generalization of the results presented in [3]. Moreover, it clarifies related results by Zaki [5] and Bell [9] in regards to the maximum number of finite transmission zeros achievable with that topology.

\section{Theory}

The two-resonator model introduced in [5] is extended to the case of $n$ resonators. A set of $n$ resonators are coupled to one another by the frequency independent coefficients $M_{i j}$. Both the source and the load are coupled to more than one resonator and possibly to each other. Since the model can be straightforwardly deduced from the discussion in [5] it is not reproduced here. The resistive terminations are set to unity.

A simple analysis of the network shows that the loop currents are given by a matrix equation of the form

$$
-j[R]+\omega^{\prime}[W]+[M][I]=[A][I]=-j[e], \quad j^{2}=-1 .
$$

Here. $[R]$ is a $(n+2) \times(n+2)$ matrix whose only non-zero entries are $R_{11}=R_{(n+2),(n+2)}=1$, 
$[W]$ is similar to the $(n+2) \times(n+2)$ identity matrix except that $W_{11}=W_{(n+2),(n+2)}=0[9]$, and $[M]$ is the $(n+2) \times(n+2)$ symmetric coupling matrix. The excitation vector is $[e]^{t}=[1,0,0, \ldots, 0]$. The low-pass prototype frequency is denoted by $\omega^{\prime}$ and is related to the actual frequency by the standard lowpass-to-bandpass transformation, i.e., $\omega^{\prime}=\omega-1 / \omega$. The transmission coefficient is given by (assuming that load and source resistors are set to unity)

$$
t=-2 j\left[A^{-1}\right]_{m 1}, \quad m=n+2 .
$$

The transmission zeros are therefore the zeros of $\left[A^{-1}\right]_{m 1}$ where the notation $m=n+2$ is introduced for convenience.

We define the $m \times m$ topology matrix $[P]$ of the network by

$$
\begin{aligned}
& P_{i j}=1 \quad \text { if } \quad M_{i j} \neq 0 \\
& P_{i j}=0 \text { if } \quad M_{i j}=0
\end{aligned}
$$

The technique presented in [3] can not be used to compute the inverse of the matrix $[A]$ in equation (1) due to the form of the matrix $[W]$. Fortunately, it is possible to manipulate this matrix and put it in a suitable form without affecting the transmission zeros.

The gist of the approach is to note that the term $\left[A^{-1}\right]_{m 1}$ can also be written as the ratio of two determinants using Kramer's rule, i.e.,

$$
\left[A^{-1}\right]_{m 1}=\frac{\text { cofactor } \mathrm{A}_{1 \mathrm{~m}}}{\operatorname{Det}(\mathrm{A})}
$$

This equation shows that the transmission zeros are the zeros of the cofactor of $[A]_{1 \mathrm{~m}}$. From the definition of this cofactor one can easily show that it does not depend on the entries $[A]_{11}$ and $[A]_{m m}$. For our purpose, this simply means that the number and locations of the transmission zeros are not affected if the first and last diagonal entries in the matrix $[A]$ are changed. A familiar consequence of this property is the independence of the transmission zeros of the resistive terminations [10, p.21]; indeed these appear only in $[A]_{11}$ and $[A]_{m m}$.

Using this property, we can set $[A]_{11}=[A]_{m m}=\omega^{\prime}$. Under these conditions, the matrix $[W]$ in equation (1) becomes equal to the $m \times m$ identity matrix; the Soriau-Frame algorithm used in [3] can now be used to show that:

Theorem: the maximum number of finite transmission zeros generated by a topology matrix $[P]$ is given by $n_{z}=n+1-k$ where $k$ is the smallest integer for which $\left[P^{k}\right]_{m 1}$ is non-zero. Here, $\left[P^{k}\right]$ is the kth power of the topology matrix $[P]$.

The proof of this result follows the discussion in [3] and is not presented here.

\section{Examples}

We first consider the case when the source is coupled to the load such that $[P]_{m 1} \neq 0$. In this case the theorem shows that the maximum number of transmission zeros is $n_{z}=n+1-1=n$, i.e., equal to the number of resonators. The two-resonator filter with two transmission zeros presented in [5] is an example of this case.

A 3-resonator filter with 2 transmission zeros was designed and measured by Liang and Blair [4]. A similar filter was also designed by Rosenberg and Hägele [8]. The topology of theses two designs is given by the matrix (assuming synchronously tuned resonators)

$$
P=\left[\begin{array}{lllll}
0 & 1 & 0 & 1 & 0 \\
1 & 0 & 1 & 0 & 0 \\
0 & 1 & 0 & 1 & 0 \\
1 & 0 & 1 & 0 & 1 \\
0 & 0 & 0 & 1 & 0
\end{array}\right]
$$

Since $[P]_{m 1}=[P]_{51}=0$ we examine $\left[P^{2}\right]_{51}$. A simple multiplication shows that this term is $\left[P^{2}\right]_{51}=1$. It therefore follows that the number of zeros of this network is $n_{z}=3+1-2=2$. This result is experimentally confirmed in [4], Fig. 14. and [8]. Fig. 4. 
Rosenberg and Hägele also presented filters of orders 5 and 6 with 4 finite transmission zeros each [8]. The topology matrix corresponding to the 5 th order filter is given by

$$
P=\left[\begin{array}{lllllll}
0 & 1 & 0 & 0 & 0 & 1 & 0 \\
1 & 0 & 1 & 0 & 1 & 0 & 0 \\
0 & 1 & 0 & 1 & 0 & 0 & 0 \\
0 & 0 & 1 & 0 & 1 & 0 & 0 \\
0 & 1 & 0 & 1 & 0 & 1 & 0 \\
1 & 0 & 0 & 0 & 1 & 0 & 1 \\
0 & 0 & 0 & 0 & 0 & 1 & 0
\end{array}\right]
$$

Direct application of the theorem to this topology matrix gives $[P]_{71}=0$ and $\left[P^{2}\right]_{71}=1$. Therefore, the maximum number of transmission zeros achievable by this arrangement of resonators is $5+1-2=4$, a result confirmed in [8], Fig. 3. The application of the algorithm to the 6 th order filter shown in [8] as Fig. 5 also predicts the observed number of finite transmission zeros. The validity of the theorem was also confirmed by testing filters of higher orders which were subsequently synthesized to confirm the anticipated number of transmission zeros.

\section{Conclusions}

A theorem which determines the maximum number of transmission zeros which can be generated by $n$ coupled resonators with a given topology matrix and where the source or load is coupled to more than one resonator was presented.

Acknowledgement: The authors would like to thank Uwe Rosenberg of Bosch Telecom, Germany for fruitful discussions.

\section{References}

[1] R. G. Cameron, "General coupling matrix synthesis methods for Chebychev filtering functions", IEEE Trans. Microwave Theory Tech., Vol. 47, pp. 433-442, April 1999.

[2] A. Atia and Williams, "New type of waveguide bandpass filters for satellite transponders", COMSAT Tech. Rev.. Vol. 1, pp. 21-43, 1971.

[3] S. Amari, "On the maximum number of finite transmission zeros of coupled resonator filters with a given topology", IEEE Microwave Guided Wave Lett., Vol. 9, pp. 354-356, Sept. 1999.

[4] J. F. Liang and W. D. Blair, "High-Q TE01 mode DR filters for PCS wireless base stations". IEEE Trans. Microwave Theory Tech., Vol. 46, pp. 2493-2500, Dec. 1998.

[5] K. A. Zaki, C. Chen and A. Atia, "Modeling of coupling probes in dual mode cavities ", IEEE MTT-S Int. Symp. Dig., pp. 515-518, 1988.

[6] B. Easter and J. Powell, "Direct-coupled resonator filters with improved selectivity". IEE Electron. Lett., Vol. 4, no. 19, p. 415 Sept. 1968.

[7] P. Röschmann, " Compact YIG banspass filter with finite-pole frequencies for applications in microwave integrated circuits", IEEE Trans. Microwave Theory Tech., Vol. MTT-21, pp. 52-57, Jan. 1973.

[8] U. Rosenberg and W. Hägele, " Advanced multimode cavity filter design using source/load-resonance circuit cross couplings", IEEE Microwave Guided Wave Lett., Vol. 12, pp. 508-510, Dec. 1992.

[9] H. C. Bell Jr., " Canonical asymmetric coupled-resonator filters", IEEE Trans. Microwave Theory Tech., Vol. MTT-30, pp.1333-1340, Sept. 1982.

[10] G. L. Matthaei, L. Young and E. M. T. Jones, Microwave filters, impedance matching networks and coupling structures, Artech House, Dedham: 1980. 